\title{
EMBEDDED SYSTEMS ENSURING SAFETY FOR PEOPLE WITH DISABILITIES
}

doi:10.2478/czoto-2021-0031

Date of submission of the article to the Editor: 06/12/2020

Date of acceptance of the article by the Editor: 24/03/2021

Tomasz Chajduga ${ }^{1}$ - orcid id: 0000-0001-6342-7210

${ }^{1}$ Czestochowa University of Technology, Poland

Abstract: The publication discusses the concept of modern embedded systems that can be programmed and build in bigger systems in order to ensure the safety of people with disabilities. Article presents the definition of embedded system and, also, introduces the definition of the author. What is more, article briefly presents the most popular and developed in the fastest way exemplary systems: ARM, STM32, Raspberry PI, Sony Spresense and NvidiaJetson. Moreover, the main reasons were described why the embedded systems are used in more and more solutions and the factors that strengthen this trend have been noted: labor costs reduction through automation, reduction of costs of development of a new product through the possibility of making quick changes and reduction of product testing time, value creation potential and, also, the way to individualize the possibilities of electronics. Also, the examples of usage of those systems were presented. Finally, the practical side of programming and testing the embedded systems were described: 'radar' (a torch) for blind people, help with balance disorders and the device to help with problems with orientation in space. In the conclusions, author shapes the estimated potential future of electronics supported with modern embedded systems, which as author believes makes this article the comprehensive study.

Keywords: embedded systems, disability, ensuring safety, programmable prostheses, intelligent prosthetics.

\section{INTRODUCTION}

According to Omni Sci website, an embedded system is a microprocessor-based computer hardware system with software that is designed to perform a dedicated function, either as an independent system or as a part of a large system. At the core is an integrated circuit designed to carry out computation for real-time operations. Besides the official definition, the author would like to present his own definition: An electronic chip that is possible to be programmed and, as a result of programming, is possible to control other, generally much larger, system or systems. The introducing own definition is important to the author, because, despite the simplicity, it understands the embedded system just as a chip, not a computer system itself. This tiny difference, according the author is relevant, because in nowadays world, for example, when talking about electronic calculator or electronic quartz hand watch (not a smartwatch!), not many people think about computer. In past, any electronic device 
that had capabilities to count and be programmable in some tiny part was perceived as computer system. Now it is the other way. That is why the author presented his own definition and believes it it is more suited to our times: the embedded system understood as a chip that can be a part of computer, but does not have to. That chip can be simply a controller or, to be more specific, a micro-controller. The review of new technology and methods can by find in some scientific works (Alavi and Leidner, 1999; Buhalis and Law, 2008; Daugherty, et al., 2005; Klimecka-Tatar and Ingaldi, 2020; Schwenk, 1986; Thompson and Bee, 2000; Wright, et al. 1998).

\section{METHODOLOGY OF RESEARCH - THE NOWADAYS EMBEDDED SYSTEMS}

In the beginning, it must be emphasized that there has been developed very many embedded systems on the market by now. The reason of such situation is high level of specialization of embedded system that can be obtained at reasonable cost. Now, embedded systems (also called micro-controllers) are common to be used in many places. The great examples are: control panels in cars, vehicle infotainment systems, air condition control panels, and so on. The challenge was to design one embedded system that could serve in many places. It happened, for example the very same electronic chip could be built in car infotainment system and electronic books reader. However, the last years brought the new trends in embedded systems that are easier to input signals from many new types of sensors, easier to program (also with implementation of very state of the art solutions and approaches) and far more powerefficient. This is important, because there is general need to make societies feel more positively about new energy technologies, to reduce the consumption of fossil fuels in the world, not to overuse the possibilities of our planet. Above mentioned aspects enabled to design and make a very flexible in the meaning of multi-use embedded systems that have significantly high potential to recreate our reality. The exemplary main embedded systems that fulfill the above conditions have been presented below.

\subsection{The examples of embedded systems based on ARM}

ARM is the abbreviation of ARM Holdings PLC, the British company that develops the ARM architecture, which stands for Advanced RISC Machine, (originally in past called Acorn RISC Machine) - 32-bit and 64-bit architecture of RISC processors. Various versions of ARM cores are widely used in embedded systems. They are characterized by low power consumption through entire energy-saving architecture, implemented multi-threading mechanisms, with the possibility of using the TCP / IP stack contained in the system or a file system (eg FAT32). ARM-based processors are one of the most widely used processors in the world. They are used, among others, in hard drives, cell phones, routers, calculators and even in children's toys. In 2005, nearly 1.7 billion processors based on ARM technology were produced, which accounted for $75 \%$ of all 32 -bit processors on the market. From the software-side point of view various specifically designed operating systems can be installed on ARM-based solutions: Windows CE, FreeBSD, NUTOS (Ethernut), Embedded Debian Linux, Embedded Ubuntu Linux and more.

\subsection{STM32}

STM32 is a family of 32-bit microcontroller integrated circuits produced by STMicroelectronics. The STM32 chips are grouped into related series that are based around the same 32-bit ARM processor core. Each product in series is specified to be 
for example more powerful or more energy saving, designed to be suitable to concrete solution. From the legal perspective, ST Microelectronics licenses the ARM Processor IP from ARM Holdings, presented above. The ST company uses the core design delivered by ARM in the ST individual configuration. It can be said that ST Microelectronics develops the core idea of ARM. This development of this idea is easy to observe in ST own peripherals attached to its products.

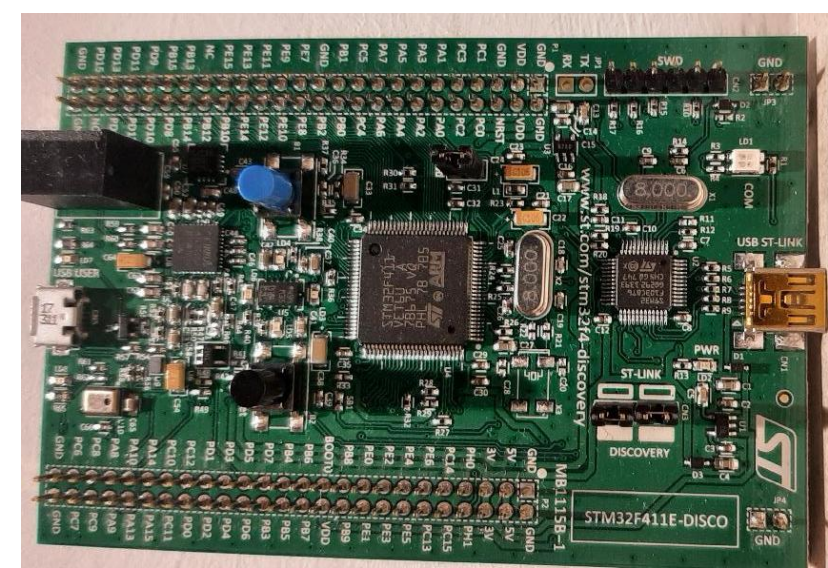

Fig. 1. The STM32 solution with ARM chip on development discovery board

\subsection{Raspberry PI}

Unlike the previously presented solutions, Raspberry $\mathrm{PI}$ is a true computer system. Despite the Broadcom BCM2835 chip embedded system, the Raspberry $\mathrm{PI}$ is equipped with USB ports, RJ45 connector, has the HDMI port and is able to connect via WIFI. It is also worth to mention that this platform has comparable to ordinary computers amount of RAM memory (up to 8GB RAM in December, 2020) There is even the do-it-yourself project transforming the Raspberry PI system into a laptop. Moreover, after the installation of one of the dedicated operating systems (Raspberry PI OS, Windows IOT, Linux Ubuntu) it is possible to use it like common, ordinary computer machine with graphical interface. In contrary, Raspberry PI is equipped in GPIO ports. GPIO stands for General Purpose Input/Output ports. This set of connections enables Raspberry to cooperate with many sensors and controlled devices. It is possible to run emulators of many other systems and work as a platform for old games, hard to be run on modern computers.

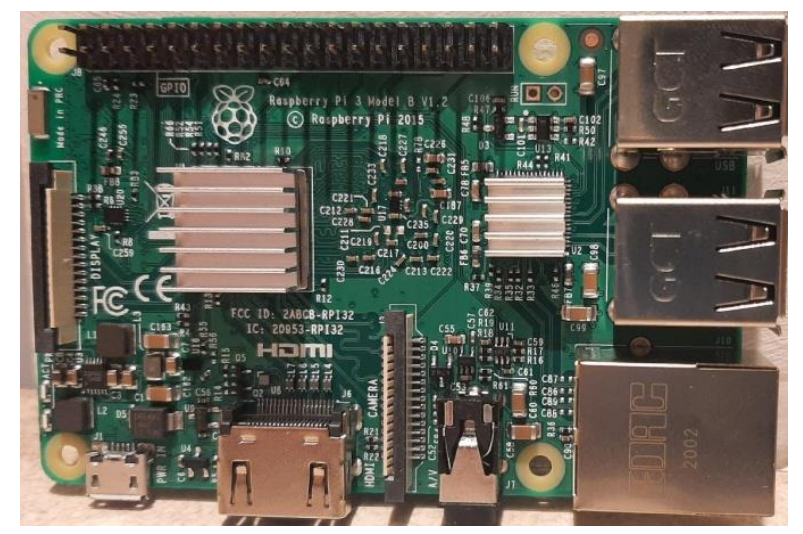

Fig. 2. The Raspberry PI computer with radiators mounted 


\subsection{Sony Spresense}

The embedded system delivered by Sony is a compact development board based on a Sony CXD5602 microcontroller with an ARM Cortex M4F Hexa-Core 156 MHz core. The microprocessor has an integrated GNSS system supporting GPS, QZSS and Glonass. Built-in advanced 192kHz / 24-bit audio codec and amplifier for audio output and support for output channels up to 8 microphones. The module also has an input for a dedicated camera.

\subsection{NvidiaJetson}

The NvidiaJetson solution is a series of embedded computing boards from Nvidia. The Jetson TK1, TX1 and TX2 models all carry a Tegra processor (or SoC) from Nvidia that integrates an ARM architecture central processing unit (CPU). Jetson is a low-power system and is designed for accelerating machine learning applications. The NvidiaJetson stands out with high compute possibilities thanks to comparably powerful microprocessors. Those systems are also equipped with many GPIO ports and highly efficient radiators. On the other hand, they use more power than competitive solutions. NvidiaJetson is most often used in places where comparably large amount of data must be processed instantly or no even minor delay is allowed.

\section{RESULTS - THE MULTITUDE OF REASONS FOR EMBEDDED SYSTEMS}

There are many reasons for embedded systems to be used in the development projects as well as in many industries in the economy. Modern economy requires fast, effective and power-efficient solutions. That is why, more and more companies needs unified technologies, designed in the way that is scalable and easy to transform, supporting automation and with huge potential for cost reduction. In points, the main reasons for usage the embedded systems are as following:

- Labor costs reduction through automation,

- Reduction of costs of development of a new product through the possibility of making quick changes and

- Reduction of product testing time,

- Value creation potential,

- A way to individualize the possibilities of electronics.

\subsection{Labor costs reduction through automation}

First of all, the embedded systems support automation. Automation is desired because of need to keep the labor costs on reasonable level in the companies. Modern embedded systems are flexible and support usage of many possible sensors and cameras as well as many output devices. Individually selected inputs and outputs give room to steer the processes automatically, with high precision and on time.

\subsection{Reduction of costs of development of a new product through the possibility of making quick changes and reduction of product testing time}

This aspect follows directly from the fact that GPIO ports can be independently programmed. Changing the assumptions taken under consideration when designing new product can drastically change the level of costs. Through the usage of embedded system many companies can use its flexibility and the fact that embedded system can be easily programmed. Potentially, in many cases in order to have a new product, having new functions it is enough to reprogram the unit or add one or more 
input/output peripherals. There is no need to redesign the product itself. Moreover, the result of changes made in the described above way is far more predictable that in case of redesigning the entire product. Therefore, the costs are more predictable and the management is easier. Product is tested faster without necessity to found out the new testing procedures, because its weak spots are well known and its nature is already understood.

\subsection{Value creation potential}

The embedded systems literally encourage to play with new sensors, new programs and new solutions. The input/output peripherals are very diversified from the functional perspective but quite unified from the electrical point of view. The result is that it is comparably easy to implement new vision for the system to work. Moreover, the programming platform is quite open, enabling to use many libraries and drivers (software controllers). To implement technology basing on neural networks or artificial intelligence (machine learning) is almost as simple as implementing the library enabling the sound emission. In the opinion of the author, the example above shows clearly the scope of potential of embedded systems.

\subsection{A way to individualize the possibilities of electronics}

The last pointed out aspect, way to individualize the possibilities of electronics, will be discussed separately in the following part of this paper.

\section{DISCUSSION - THE USAGE OF MODERN EMBEDDED SYSTEMS}

There are many examples, where the modern embedded systems can be used for. Taking under consideration their flexibility and openness for the newest technologies it is hard to list all the ways that they could be potentially used. Therefore, the presented below list is very imperfect. The author perceives following exemplary using of modern embedded systems: management support, smart home, automation, measurement, safety support systems (e.g. in vehicles), ensuring safety for people with disabilities and many other.

\subsection{The exemplary usage in general purposes}

There are many examples, where the modern embedded systems can be used for. Taking under consideration their flexibility and openness for the newest technologies it is hard to list all the ways that they could be potentially used. Therefore, the presented below list is very imperfect. The author perceives following exemplary using of modern embedded systems: management support, smart home, automation, measurement, safety support systems (e.g. in vehicles), ensuring safety for people with disabilities and many other.

\subsection{The usage in specific purpose of ensuring safety for people with disabilities} While talking about safety, the specific needs should be recognized and the scope of help that is possible to obtain by a nowadays embedded system usage should be analyzed. The question of the main purpose how to ensure safety for the disable person via use of micro-controller must be considered with regard to the following aspects:

- individually (related to the type of disability) programmed inputs and outputs of the embedded system; 
- individually (related to the type of disability) chosen sensors and output devices;

- assumed durability of the system and its instant helping readiness time (the issue of power consumption and weight of the battery itself).

The following examples will be considered with regard to the aspect of usage in specific purpose of ensuring safety for people with disabilities.

\subsection{Example 1 - 'radar' (a torch) for blind people}

In order to design the radar solution for blind people that could take a shape a form of specific torch the following configuration of inputs and outputs is proposed:

- Input: ultrasonic sensor

- Output 1: sound notification,

- Output 2: motor generating vibrations (warning vibrations).

The ultrasonic sensor is required in order to get the information about the distance between the header of the torch and the obstacle. The signal received by the sensor must be transformed (processed) by the embedded system in order to control the buzzer or speaker (sound notification) or electric motor (vibrations). The team designing the device assumes that 3 modes will be enough to satisfy the needs of the market. First mode called "Be normal" is basing on simple sound notification as a series of beeps the more intensive the closer to the obstacle and becoming continuous sound when the obstacle is within reach. This mode is most suitable for blind people that are also hearing impaired due to clear sound emitted. Second mode called "Be silent" is being designed for situations when beeping notification could be disturbing for others (f. ex. in church), discretion is expected or where sound notification could not be heard (f. ex. very loud street). The third mode is emulating the Star Wars Jedi sword sound and is called "Be cool". This mode is been designed to young users and generally for people with sense of humor.

\subsection{Example 2 - help with balance disorders}

In order to design the helping device with balance disorders, the solution could take a form of a machine recognizing the problems with keeping balance and mass manipulation module. The following configuration of inputs and outputs is proposed:

- Input 1: gyroscope,

- Input 2: accelerometer (acceleration sensor),

- Output 1: mass manipulation servo,

- Output 2: sound notification,

The second example assumes two inputs and two outputs. However, this can be modified, if need. In the basic version of the device it is equipped with gyroscope and accelerometer that are able to pass the information about position and movement of disable person to the embedded system. The chip would process the data and, through the output, steer the mass manipulation mechanism, helping the person to keep the balance. Additional optional sound notification can be useful to signalize the moment of possible loss of balance. It is worth to mention that the system, depending on the complexity of used algorithms, could not only simply recognize the moment of loosing balance but also, throughout Al and machine learning, intelligently anticipate it. 


\subsection{Example 3 - help with problems with orientation in space}

In order to design the device helping with problems with orientation in space, the following configuration of inputs and outputs is proposed:

- Input 1: GPS module (through special protocol, f. ex. I2C)

- Input 2: magnetic field sensor

- Input 3: data from server (f. ex. Google Maps)

- Output 1: speaker (to have voice notifications where to go),

- Output 2: mechanical 'screen' 'presenting' or so-to-say reflecting the map (surroundings) on the mechanical device - that blind or partly blind person may realize where he or she is.

The general scheme of working is similar to the previously presented examples. However, in this case the data transformation process is significantly more complex and requires better computing power as well as higher level of data virtualization. The result can be characterized by far better representation of the surroundings for blind people.

\subsection{Practical aspects of programming the embedded systems to improve the safety level of people with disabilities}

In order to program the micro-controller, special software must be installed on the PCcompatible computer. The example of such software is STM32Cube. The picture below presents the part of this software. Embedded programming bases on the setting the pins of the micro-controller as "in" pins (for imputing signals) or "out" pins (for passing transformed signals). Moreover, not all pins can be used for all purposes, therefore often more technical knowledge is needed. The next step is to program the pins. Picture below shows the fragment of the program in the software compatible with STM32 embedded solutions. In order to verify the accuracy of the programming, the embedded system must be connected to the real electronic components and tested. The example presented in following picture is rather simple. It must be stated that inputs as well as outputs can be highly diversified. The last phase is preparation to mass manufacturing. This phase enables to mount the embedded system in its working environment They are car safety solutions, car infotainment centers, toys, measuring tools, smart home solutions and many more, as mentioned before.

\section{CONCLUSIONS}

In modern embedded systems great variety of technologies can be connected in order to cooperate and product the stunning results. The most interesting, according to author, are the following: ultrasonic sensors, cameras, emergency power supplies, $\mathrm{SSH}, \mathrm{RC}$, python libraries introducing the micro-controllers into the world of machine learning (also called the $\mathrm{Al}$ - artificial intelligence), advanced image processing software and more.

As a part of a conclusion, the author would like to emphasize the enormous potential of embedded systems for a building many elements of future world. Gathering together and final analysis of above presented information can result in following conclusions:

- The trends are that electronics will become more and more individualized;

- Access to personalized electronics is getting cheaper and cheaper;

- Systems ensuring the safety of people with disabilities are becoming more and more accessible. 
In terms of peoples wit disabilities, the next step (with high probability) will be individually programmed prostheses and the development of programmable, intelligent prosthetics. The author predicts the revolution in prosthetics, significant improvement in comfort and quality of life of disabled people.

\section{REFERENCES}

Alavi, M., Leidner, D.E. 1999. Knowledge management systems: issues, challenges, and benefits, Communications of the AIS, 1.

ARM Webpage, https://www.arm.com/, (accessed: 02.12.2020)

A Survey on Optimized Implementation of Deep Learning Models on the NVIDIA Jetson Platform, https://www.researchgate.net/publication/329802520_A_Survey _onOptimized_Implementation_of_Deep_Learning_Models_on_the_NVIDIA_Jetso n_Platform(accessed: 10.12.2020)

Buhalis, D., Law, R. 2008. Progress in information technology and tourism management: 20 years on and 10 years after the Internet - The state of eTourism research, Tourism management 29 (4), 609-623.

Daugherty, P.J., et al. 2005. Reverse logistics: superior performance through focused resource commitments to information technology, Transportation Research Part E: Logistics and Transportation Review 41 (2), 77-92.

Edge computing with low power consumption, https://developer.sony.com /develop/spresense/, (accessed: 11.12.2020)

Embedded systems, Omni Sci, https://www.omnisci.com/technical-glossary/ embedded-systems, (accessed: 11.12.2020)

KAMAMI oficjalnym partnerem STMICROELECTRONICS, https://stm32.eu/, (accessed: 10.12.2020)

Klimecka-Tatar, D., Ingaldi, M., 2020. People's Attitude to Energy from HydrogenFrom the Point of View of Modern Energy Technologies and Social Responsibility, Energies, Basel, Switzerland: MDPI.

NVIDIA Jetson TX2 Tegra Developer Kit, https://www.precisioncomputers. com.au/nvidia-jetson-tx2-tegra-developer-kit/, (accessed: 11.12.2020)

Raspberry Pi 400 - Your complete personal computer built into a compact keyboard, https://www.raspberrypi.org/, (accessed: 10.12.2020)

Raspberry Pi GPIO pins., https://forum.arduino.cc/index.php?topic=539419.0, (accessed: 11.12.2020)

Schwenk, Ch. H. 1986. Information, cognitive biases, and commitment to a course of action, Academy of Management Review 11 (2), 298-310.

Thompson, T., Bee, L. T., 2000. Information systems orientation and business use of the Internet: An empirical study, International Journal of Electronic Commerce 4 (4), 105-130.

Valvano, J.W., Embedded systems: introduction to arm cortex-m microcontrollers http://users.ece.utexas.edu/ valvano/arm/outline1.htm, (accessed: 11.12.2020)

Wright, P. M., Scott A. Snell. 1998. Toward a unifying framework for exploring fit and flexibility in strategic human resource management, Academy of management review 23 (4), 756-772. 